\title{
Development and evaluation of the Clinician Suicide Risk Assessment Checklist
}

\author{
Robert King $^{1,2}$, Chris Lloyd ${ }^{3}$, Tom Meehan ${ }^{2}$, Khaleda O'Neill ${ }^{3}$ and Cindy Wilesmith ${ }^{3}$ \\ 1. Department of Psychiatry, The University of Queensland, Brisbane, Australia \\ 2. The Park Centre for Mental Health, Brisbane, Australia \\ 3. Division of Occupational Therapy, The University of Queensland, Brisbane, Australia
}

\begin{abstract}
This paper describes the development and evaluation of a new instrument - the Clinician Suicide Risk Assessment Checklist (CSRAC). The instrument assesses the clinician's competency in three areas: clinical interviewing, assessment of specific suicide risk factors, and formulating a management plan. A draft checklist was constructed by integrating information from 1) literature review 2) expert clinician focus group and 3) consultation with experts. It was utilised in a simulated clinical scenario with clinician trainees and a trained actor in order to test for inter-rater agreement. Agreement was calculated and the checklist was re-drafted with the aim of maximising agreement. A second phase of simulated clinical scenarios was then conducted and inter-rater agreement was calculated for the revised checklist. In the first phase of the study, 18 of 35 items had inadequate inter-rater agreement $(60 \%)>$, while in the second phase, using the revised version, only 3 of 39 items failed to achieve adequate inter-rater agreement. Further evidence of reliability and validity are required. Continued development of the CSRAC will be necessary before it can be utilised to assess the effectiveness of risk assessment training programs.
\end{abstract}

Keywords

suicide, suicide risk assessment, clinician training, instrument development, evaluation

\section{Introduction}

Globally suicide is a major health problem. A recent Queensland based survey instigated by The World Health Organization (WHO) estimated that $10.4 \%$ of the population seriously consider suicide at some point in their lifetime while approximately $4.2 \%$ attempt suicide (De Leo, Cerin, Spathonis \& Burgis, 2005). WHO has prioritised the reduction of suicide as one of its primary goals (Rutz, 2001). In Australia, suicide, particularly youth suicide, has long been prioritised on the public health agenda. In 1999 the government released the National Action Plan for Suicide Prevention, and in 2000 the Living is for Everyone (LIFE) framework for the prevention of suicide and self-harm (Commonwealth Department of Health and Ageing, 2000). These were devised to guide future reforms addressing suicide prevention. As of July 2005, the Commonwealth was supporting seven projects of national importance that had suicide prevention as a primary aim (Commonwealth Department of Health and Ageing, 2006). WHO advises that a significant reduction in suicide is attainable if appropriate treatment is provided (Rutz, 2001).

\footnotetext{
Contact: $\quad$ Dr. Chris Lloyd, Division of Occupational Therapy, The University of Queensland, St Lucia, Queensland 4072 c.1loyd@shrs.uq.edu.au

Citation: $\quad$ King, R., Lloyd, C., Meehan, T., O’Neill, K. \& Wilesmith, C. (2006). Development and evaluation of the Clinician Suicide Risk Assessment Checklist. Australian e-Journal for the Advancement of Mental Health 5(1) www.auseinet.com/journal/vol5iss1/king.pdf

Published by: Australian Network for Promotion, Prevention and Early Intervention for Mental Health (Auseinet) www.auseinet.com/journal Received 6 November 2005; Revised 16 May 2006; Accepted 16 May 2006
} 
This position is justified by research indicating that a substantial number of suicide victims do contact health services some weeks, months or years before their suicide (Barraclough, Bunch, Nelson \& Sainsbury, 1974; Cheng, 1995; Isacsson, Bergman \& Rich, 1994; Isometsa, Henriksson, Aro et al., 1994; Rihmer, 1996). By drawing on an extensive body of suicide research, which has consistently and convergently identified factors that are associated with suicide and suicidal behaviour, practitioners are able to make informed judgements about individual clients' suicide risk (Commonwealth Department of Heath and Ageing, 2000).

Suicide risk assessment is a key competency required by mental health professionals when working with suicidal clients. This key competency has been reflected in policies and practice, both overseas and in Australia (Doyle, 2003; Schwartz \& Rogers, 2004). Risk assessment skills are identified in the National Practice Standards for the Mental Health Workforce (Commonwealth Department of Health and Ageing, 2002) as a required key competency for all mental health workers.

State governments have also been active in suicide prevention. The Victorian Government allocated $\$ 1.7 \mathrm{~m}$ to suicide prevention training (Victorian Department of Human Services, 2003). The Queensland Government Suicide Prevention Strategy 2003-2008 outlines current key initiatives and identifies increasing the knowledge and skills among services and facilitating appropriate training and education for early intervention as key priorities (Queensland Government, 2003). Other states have also developed initiatives designed to enhance professional and non-professional responses to suicide risk, and Applied Suicide Intervention Skills Training (ASIST) has been implemented widely by LivingWorks, in association with Lifeline (see www.livingworks.net/ASX.php ).

While it is difficult within populations of people receiving treatment for mental health problems to identify demographic or service factors that will identify those at greatest risk (Pirkis, Burgess \& Jolley, 2002), people who subsequently commit suicide frequently seek help prior to suicide and it is important that clinicians are able to engage with such people and identify immediate risk factors and clinical treatment needs (American Psychiatric Association, 2003).
The effectiveness of training designed to increase the competency of mental health professionals in working with suicidal clients is largely unquantified. Indeed the literature in the area of assessing training effects of suicide risk assessment and management training programs is somewhat controversial. Training effects are not consistently found in all studies. It is argued that this may be the result of inconsistency of methods used to assess training effects. Currently, there is no single agreed upon gold-standard for assessing training effects. The development of an assessment instrument to measure the effectiveness of suicide risk assessment and training is therefore likely to assume importance. This could be in relation to demonstrating competencies in training and accreditation of suicide prevention programs.

\section{Assessment of training effects in suicide risk assessment and management programs}

In the only Australian study, by Simpson, Winstanley and Bertapelle (2003), a New South Wales suicide prevention training program was modified so it would be specifically appropriate for intervention and assessment of clients with traumatic brain injury. Although a training effect was found the methods of assessment make the usefulness of these results questionable. The study utilised only subjective selfassessment inventories of changes in knowledge and attitude, and an objective knowledge test. Other studies to find a training effect when using unstandardized self-assessments include Doyle (2003) and Juhnke (1995). This is problematic since although changes in knowledge and selfperceived changes in skills and attitudes may be of interest, they do not demonstrate the trainee's clinical skills and application of knowledge. It is argued in the literature that using vivo assessments is the only method to provide truly objective and valid assessment of trainees' performance in suicide assessment and management (Morriss, Gask, Battersby et al., 1999; Tierney, 1994).

A UK study by Fenwick, Vassilas, Carter and Haque (2004) also fails to reach this ideal form of assessment by applying The Suicide Response Inventory II (SRI-II) to its assessment of training effects. The SRI-II is a 25 item, self-administered questionnaire comprising a series of hypothetical client 
remarks followed by two possible 'helper' replies, one of which is considered facilitative for suicide prevention whereas the other is either neutral or deleterious. This test has been found to have adequate construct validity as measured by its ability to discriminate between three groups of respondents known to differ in suicide counselling skills i.e. masters level psychology students and untrained psychology students. Discriminate validity tested using the MarloweCrowne Social Desirability Scale was also found to be sound, as was test-retest reliability (Neimeyer \& Bonnelle, 1997).

Fenwick et al. (2004) found that following a risk assessment training program which included both lectures and group work with actors roleplaying clients, trainees assessed using the SRIII did exhibit a marked training effect. Whilst this could reflect the educational benefits of the course, it could also be argued that this result is due to the assessment's lack of valid measure. The question of whether performance on such a pencil and paper test reflects actual clinical performance in a clinical interview situation, as opposed to knowledge of correct clinical performance, is certainly debatable. What one can achieve under test conditions, with time to reflect upon answers, and the capacity to revise responses, may indeed be quite different from reactions in a clinical situation. Morriss et al. (1999) and Tierney (1994) argued that the most valid measure will always be a vivo assessment of skills.

One attempt has been made to develop a vivo assessment method and tool for this area. Morriss et al. (1999) developed two simple criterion scales for assessing trainees' performance. The first assessed clinical interviewing skills and was based on a scale used to assess the performance of family practitioner trainees in the assessment and management of depression (Gask, Williams \& Harrison, 1995). The second scale was independently devised by the authors and was designed to assess specific suicide risk assessment and management skills. A clear training effect was found for this group of nonmental health professionals and volunteers on a number of items on the risk assessment and management scale, but not the clinical interview scale. The authors suggested that the training may have been too short to induce changes in these kinds of skills, suggesting it may take up to six months of training to make changes in this area. However these results must be viewed with caution owing to problems with the assessment of the program.

The clinical interview scale involved conducting an utterance analysis of the first five minutes of a simulated clinical interview. This technique has proven interrater reliability, with $88 \%$ agreement between raters found in Gask, Goldberg, Lesser and Millar (1988) and a range of 63 to $70 \%$ agreement amongst different items in Morriss et al. (1999). However, this remains the only evidence of the psychometric properties of this test. Also it appears the original authors devised the test items based entirely upon their own expertise in the field. No reference was made to any qualitative or quantitative research undertaken to generate the test items. The validity of this test seems questionable.

The second criterion scale specifically measured suicide risk and management skills. Items were based on what the authors believed to be the most clinically important microskills for assessing and managing suicide, and those which failed to achieve high inter-rater reliability (less than 60\%) were dropped from the analysis. Skills included: adequate problem solving, followup, eliciting suicidal ideas, and the provision of immediate support. As with the development of the interview skills rigorous research does not appear to have been undertaken to generate test items, and testing of psychometric properties remains limited to, what could be considered, lenient inter-rater reliability criteria $(60 \%)$.

Appleby, Morriss, Gask et al. (2000) adopted Morriss et al.'s (1999) assessment tools in their study. Unlike Morriss et al. (1999) they included a sample of mental health professionals. This proved somewhat of a challenge for the assessment tools, with a marked ceiling effect present both before and after training in this group. It would appear these tools are unsuitable for use in assessing training effects for advanced training programs with highly experienced populations.

Suicide assessment and management training programs designed to improve clinical skills amongst mental health professionals lack a reliable and valid tool for assessing their educational efficacy. To 
ensure that clinical skills, and not merely clinical knowledge, are measured, an assessment tool should appraise in vivo simulated clinical interview. It is important this tool be sensitive to training effects in diverse populations of mental health workers, so as to demonstrate the effectiveness of advanced training for these workers.

The objective of this study was to develop a reliable and valid tool for assessing the efficacy of suicide risk assessment and management training programs designed to improve clinical skill amongst mental health professionals. The Clinician Suicide Risk Assessment Checklist (CSRAC) assesses the clinician's competency in three areas: clinical interviewing, assessment of specific suicide risk factors, and formulating a management plan. In line with current research it is designed for use within simulated clinical interviews between clinician and actor. Interrater reliability was assessed by two independent raters.

\section{Method and results}

\section{Literature review}

The literature was reviewed in the three areas of competency (clinical interviewing, assessment of specific suicide risk factors, and formulating a management plan). Medline, Cinahl, PsycINFO, and Cochrane databases were searched using the search terms: 'suicide' and 'risk', 'suicide' and 'management', 'suicide' and 'prevention', 'suicide' and 'assessment' and 'suicide' and 'intervention'. Reference snowballing was used in key articles to obtain original studies. For 'assessment of specific suicide risk factors' inclusion of articles was limited to original studies with comparison control groups whilst reviews, other than systematic reviews, were excluded. This was applied in order to produce an empirically sound assessment tool. Studies observed correlations of risk factors with either completed suicide or a medically serious attempt (i.e. one which requires emergency hospitalisation followed by a period of intensive care or an attempt which in most cases results in successful completion, such as gun shot). Relevant articles were narrowed to twenty-four key studies and risk factors for suicide were identified. Table 1 indicates risk factors, study design of the source article, and the corresponding item number on the tool generated from this study, the CSRAC.
From the literature suicidal ideation, plans and preparations, and prior suicide attempt were the factors most highly associated with suicide, prior attempts being the most predictive stable factor and plans and preparations the most predictive acute factor. Assessment of ideation includes frequency, intensity, and both wishes to live and wishes to die. Previous self-harming behaviour and previous suicide attempt were both associated with suicide, the latter being most predictive. Assessment of plans and preparations included establishing the client's level of intent, presence of concrete plan, availability of means and taking care of 'unfinished business'.

Presence of a mental illness including schizophrenia, personality disorder, anxiety disorder, schizoaffective, bipolar, and depressive disorder were present in up to $90 \%$ of suicides. Uni-polar depressive disorders were the most common. Specific symptoms associated with depression were identified in studies or current risk assessment tools; the most highly associated symptoms were hopelessness and worthlessness. Other symptoms identified were reduced appetite/weight loss, insomnia, reduced ability to experience pleasure, poor concentration and memory, and reduced libido.

Factors related to family history were associated with suicide; family suicidality was recorded as the most highly correlated risk factor. Others included family psychiatric history, family relationship disturbance, and childhood abuse/neglect. The recent suicide of somebody close, for example a friend, was also associated with risk.

Personal states and traits associated with risk of suicide were impulsivity, social isolation, physical illness, hostility, and drug and alcohol dependence. A risk factor of social isolation was recorded in the presence of divorce, not married, living alone, or subjective ratings of a reduced social network. The most common physical illnesses associated with risk were either chronic or terminal. The presence of a stressful life event was highly associated with risk of suicide. Common stressors included legal, financial, employment, family, and interpersonal problems or loss. 


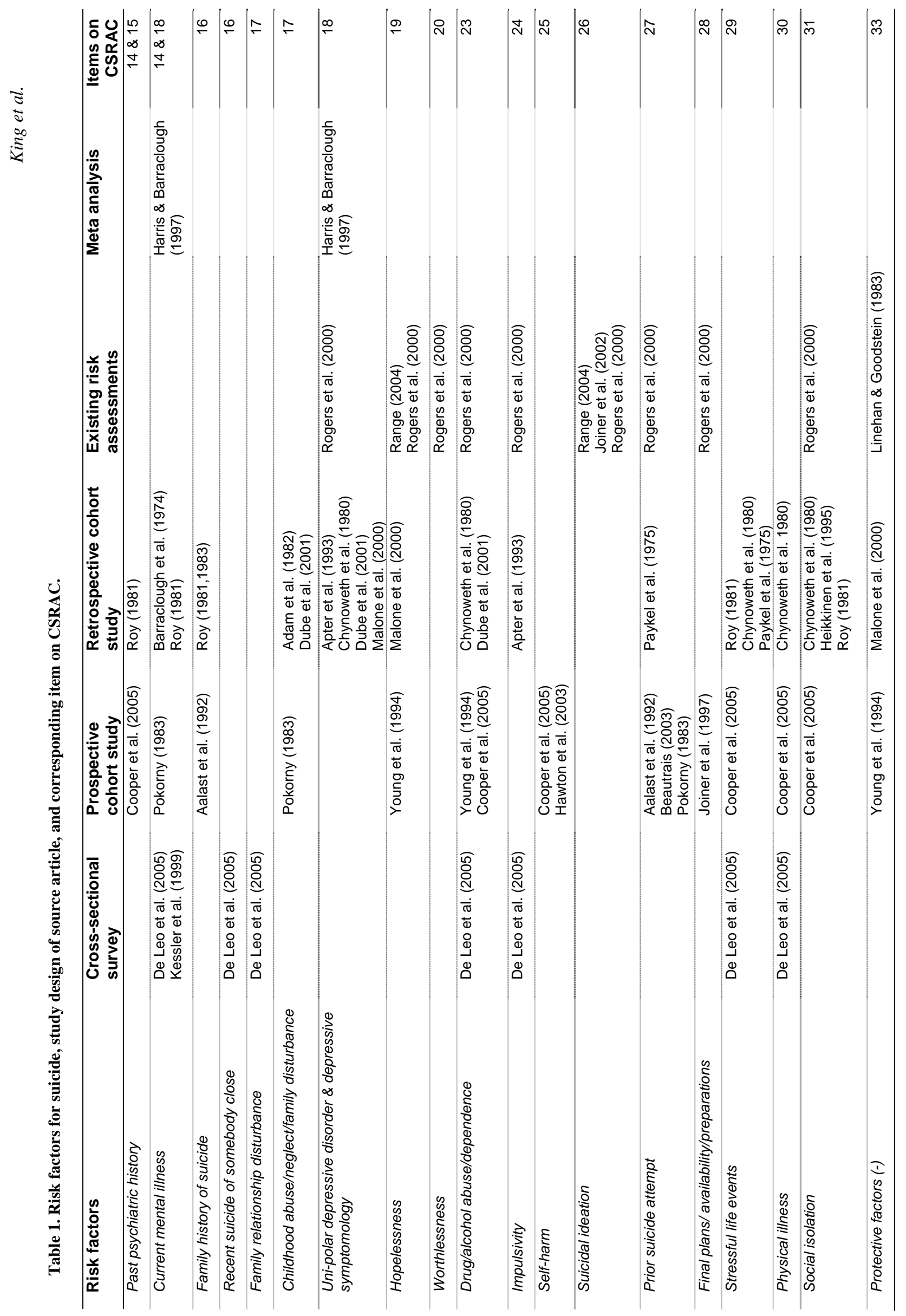


From the literature, factors that were inversely associated with suicide risk are termed reasons for living. Reasons for living may be anything that the 'at risk' client feels prevents him/her from the act of suicide. Research identifies some key reasons for living: child under 18 years living at home, negative beliefs/morals concerning suicide, fear of the act itself, and religious faith.

Given the lack of empirical research on 'clinical interviewing' and 'formulating a management plan', the review of the literature for these areas was expanded to include reviews, books, and clinical handbooks in suicide risk assessment over the last 10 years and recent basic counselling texts. The main factors obtained from this review are displayed in Tables 2 and 3.

Suicide literature emphasises the importance of developing an understanding of the client's context, building a strong therapeutic alliance, and emphasising a collaborative relationship whereby both clinician and client take responsibility and work as a team. These were stated as fundamental to obtaining accurate information during the interview and key to the success of the management plan.

Suicide literature indicates that empathetic listening and understanding is a core strategy toward achieving these during the interview and subsequent management of risk. Basic counselling texts were consulted for specific techniques in these skills.

Skills included: open body language which communicates maintained interest and encouragement (for example maintained but varied eye contact), paraphrasing and reflecting of client's feelings, encouraging non-verbals (for example 'um hum'), non-judgemental response to disclosure, and open ended questioning. The literature emphasises on-going assessment during management of risk, implementing intervention with knowledge of previous treatments already trialled, and balancing the maintenance of safety and preserving the independence of the client (e.g. hospitalising when only absolutely necessary).

Table 2. Interviewing skills during suicide risk assessment and corresponding item on CSRAC

\begin{tabular}{|c|c|c|}
\hline Interviewing skills & Author(s & Items on CSRAC \\
\hline Understanding the client's context and perspective & $\begin{array}{l}\text { APA (2003); Eddins \& Jobes (1994); } \\
\text { Meichenbaum (2005); O'Connor et al. (2004); } \\
\text { Rogers \& Soyka (2004); Simon (2004) }\end{array}$ & Marking criteria \\
\hline Building a therapeutic alliance & $\begin{array}{l}\text { APA (2003); Jobes \& Drozd (2004); Kleespies et } \\
\text { al. (1999); Meichenbaum (2005); Rogers \& Soyka } \\
(2004)\end{array}$ & All items part $A$ \\
\hline Collaborative relationship & $\begin{array}{l}\text { Eddins \& Jobes (1994); Jobes \& Drozd (2004); } \\
\text { Meichenbaum (2005); Rogers \& Soyka (2004) }\end{array}$ & 10 \\
\hline Empathetic understanding & Kleespies et al. (1999); Rogers \& Soyka (2004) & 9 \\
\hline Non-judgmental attitude & Meichenbaum (2005); Rogers \& Soyka (2004) & 8 \\
\hline Appropriate body language \& non-verbals & Nelson-Jones (2003) & $1-7$ \\
\hline Appropriate tone of voice \& language/wording & Nelson-Jones (2003) & $11-13$ \\
\hline
\end{tabular}

Table 3. Management planning during suicide risk assessment and corresponding item on CSRAC

\begin{tabular}{|c|c|c|}
\hline Management plan & Authors & Items on CSRAC \\
\hline Understanding the client's context and perspective & $\begin{array}{l}\text { APA (2003); Meichenbaum (2005); O'Connor et al. } \\
\text { (2004); Rogers \& Soyka (2004); Simon (2004) }\end{array}$ & Marking criteria \\
\hline Building a therapeutic alliance & $\begin{array}{l}\text { APA (2003); Jobes \& Drozd (2004); Kleespies et } \\
\text { al. (1999); Meichenbaum (2005); O'Connor et al. } \\
\text { (2004); Rogers \& Soyka (2004) }\end{array}$ & $35 \& 36$ \\
\hline Collaborative relationship & $\begin{array}{l}\text { Jobes \& Drozd (2004); Meichenbaum (2005); } \\
\text { Rogers \& Soyka (2004) }\end{array}$ & $35 \& 36$ \\
\hline Intensity of plan reflects level of risk & Simon $(2004)$ & 38 \\
\hline Ongoing assessment - evidence of follow-up & Simon (2004) & 37 \\
\hline Determining treatments used in previous episodes & Simon (2004) & 15 \\
\hline
\end{tabular}




\section{Focus group}

Participants consisted of 12 mental health practitioners with at least five years' experience in mental health, involvement in a crisis response environment, and clinical involvement with service users. All participants met these inclusion criteria and no exclusions were made.

Ethics approval was obtained from the West Moreton District Health Service, Queensland and the University of Queensland. The team leader of the Community Crisis Assessment Team was contacted via telephone and invited to participate in the study at a time and venue most convenient to them. The focus group was conducted at their weekly team meeting. Twelve team members participated. Two researchers moderated discussion and conducted questioning, and a third researcher recorded group non-verbals, corroboration and agreement, emotional responses, and summary of discussion.

A semi-structured interview format was used to guide and prompt discussion concerning their beliefs about aspects of good clinical practice in relation to suicide risk assessment, based on their experience. The duration of the discussion was approximately one hour and audiotape and researchers' written notes were used to record the session.

All data pertaining to the discussion were transcribed and analysed using a Consensual Qualitative Research method (Hill, Thompson \& Williams, 1997). First the data were independently coded into theoretical domains and then compared. Any discrepancies were resolved through discussion and consensual agreement. Finally categories within domains were coded independently and the process of consensual agreement was conducted again (Hill et al., 1997). The appropriateness of categorisation of data was checked and approved by a researcher who was independent of the focus group and coding process. The three domains included interviewing techniques, predictors of risk, and intervention planning.

\section{Consultation with experts}

Experts consisted of three mental health professionals with many years' experience in the field. Based on data from literature reviews and the focus group, a draft checklist was developed.
Items were determined based on level of agreement amongst focus group participants, level of corroboration between the focus group and the literature, ability of constructs to be measured by an observer, pragmatic consideration of the interviewing context, and elimination of paradigm specific counselling skills.

Preliminary suicide assessment scenarios were conducted by an expert clinician, the research team leader, and an employed and trained actor. During the role-play, the researchers performed a trial rating with the tool and analysed its utility in terms of clarity of instructions, benchmarking of items, appropriateness of wording, order of items, and use of rating scale. The research leader and assistant researcher provided advice and feedback on the face validity of the instrument. Following this method on three occasions, researchers redrafted revised versions of the CSRAC.

\section{Clinical interview role-plays}

Mental health clinicians who had undergone a half-day training workshop at The Park Centre for Mental Health (Brisbane) in suicide risk assessment and crisis intervention were invited to participate in the study in return for class credit. Fourteen clinicians completed the interview. An employed actor was recruited and trained for the role of 'at risk client' by the lead and assistant researchers.

The mental health clinicians were instructed to perform a suicide risk assessment interview, of no specified time limit, with an employed actor who would be depicting the scenario of a possibly depressed client. The actor was instructed to perform, in a standardised manner, the role of a client who was depressed and experiencing mild suicidal ideation. The actor was instructed to modify his disclosure of personal information based on the interviewing skills of the clinician. Participants were informed that the interviews were being visually recorded.

Seven of the fourteen interviews were originally observed by two researchers and rated according to the CSRAC. The percentage of agreement on items was analysed by the team and the CSRAC was revised again to improve inter-rater reliability. Revisions included clearer definitions 
of items, improving benchmarks for marking criteria, dividing items involving multiple constructs, and combining items that tested similar constructs. The rating scale for Part A was collapsed from a continuous scale into a dichotomised scale in order to more clearly define marking criteria and this was sensitive to picking up differences between interviews. The remaining seven interviews were rated independently by the two researchers with the final version of the CSRAC (see Appendix 1).

Percentage of agreement between raters was calculated for each item, to indicate trends in rating and to guide revision and development of the tool. The cut-off for acceptability of interrater agreement $(60 \%$ or above) was used as a benchmark from Morriss et al. (1999). Correlation statistics such as Kappa for dichotomous variables and intra-class for scaled items were not calculated given the small $n$ and insufficient power.

Table 4 shows inter-rater reliability for the first and second stages of clinical interviews. From the first stage, 18 of the 35 items did not meet criteria for acceptability $(60 \%>)$ whilst $17 \mathrm{did}$ $(60 \% \leq)$. During the second stage of interviews, using the revised CSRAC, only 3 items did not meet criteria for acceptability $(60 \%>)$ whilst 36 did $(60 \% \leq)$. All three items that did not meet criteria during the second stage of interviews did not meet criteria during the first stage. Overall there was an improvement in percentage of agreement on all items from stage 1 interviews (average $60 \%$ ) to stage 2 interviews (average $=$ $84.57 \%$ ), except for three items which declined in agreement but which still met criteria for acceptability. Variability in scoring also improved from a range of $0-100 \%$ on the first clinical interviews to $42.86-100 \%$ on the second clinical interviews.

\section{Discussion}

The objective of this study was to develop a reliable and valid tool for assessing clinicians' skills in suicide risk assessment and management. Both empirical research and expert clinical experiences were consulted for its development. Ensuring validity was attempted by using rigorous development procedures and assessment of face validity by expert clinicians; however this was not empirically tested in this study. Inter-rater agreement was assessed by calculating percentage agreement between two independent raters.

Scores of agreement from the first stage of clinical interviews were used to revise and develop the final draft of the CSRAC. Items either required further defining of the construct, benchmarking to establish explicit criteria for marking, breaking down of multiple constructs into more than one item, or combining items that addressed the same construct.

Table 4. Inter-rater reliability (IRR) for the first and second stages of clinical interviews.

\begin{tabular}{|c|c|c|c|c|c|}
\hline \multicolumn{3}{|c|}{ Stage 1 interviews } & \multicolumn{3}{|c|}{ Stage 2 interviews } \\
\hline Item & $\begin{array}{r}\text { IRRR } \\
\% \\
\end{array}$ & & Item & $\begin{array}{r}\text { IRR } \\
\% \\
\end{array}$ & \\
\hline 1 & 100 & & 1 & 100 & \\
\hline 2 & 85.72 & & 2 & 100 & \\
\hline 3 & 85.72 & & 3 & 85.72 & \\
\hline 4 & 85.72 & & 4 & 100 & \\
\hline 5 & 57.14 & * & 5 & 100 & \\
\hline 6 & 100 & & 6 & 100 & \\
\hline 7 & 85.72 & & 7 & 71.43 & \\
\hline 8 & 57.14 & * & 8 & 100 & \\
\hline 9 & 57.14 & * & 9 & 100 & \\
\hline 10 & 42.86 & * & 10 & 85.72 & \\
\hline \multirow[t]{3}{*}{11} & 42.86 & * & 11 & 42.86 & * \\
\hline & & & 12 & 71.43 & \\
\hline & & & 13 & 71.43 & \\
\hline \multirow[t]{3}{*}{12} & 0 & * & 14 & & \\
\hline & & & 15 & 100 & \\
\hline & & & 16 & 100 & \\
\hline 13 & 57.14 & * & 17 & 85.27 & \\
\hline 14 & 28.57 & * & 18 & 100 & \\
\hline 15 & 14.29 & * & 19 & 100 & \\
\hline 16 & 42.86 & * & 20 & 85.27 & \\
\hline 17 & 100 & & 21 & 100 & \\
\hline 18 & 71.43 & & 22 & 100 & \\
\hline 19 & 100 & & 23 & 85.27 & \\
\hline 20 & 14.29 & * & & & \\
\hline 21 & 100 & & 24 & 100 & \\
\hline \multirow[t]{2}{*}{22} & 28.57 & * & 25 & 100 & \\
\hline & & & 26 & 85.27 & \\
\hline 23 & 71.43 & & 27 & 100 & \\
\hline 24 & 42.86 & * & 28 & 71.43 & \\
\hline 25 & 57.14 & * & 29 & 42.86 & * \\
\hline 26 & 85.72 & & 30 & 71.43 & \\
\hline 27 & 85.72 & & 31 & 100 & \\
\hline 28 & 71.43 & & 32 & 71.43 & \\
\hline 29 & 42.86 & * & 33 & 57.14 & * \\
\hline \multirow{3}{*}{$\begin{array}{l}30 \\
\mathbf{3 1}\end{array}$} & 71.43 & & 34 & 71.43 & \\
\hline & 57.14 & * & 35 & 100 & \\
\hline & & & 36 & 85.72 & \\
\hline 32 & 57.14 & * & 37 & 85.72 & \\
\hline 33 & 57.14 & * & & & \\
\hline 34 & 85.72 & & 38 & 71.43 & \\
\hline 35 & 71.43 & & 39 & 100 & \\
\hline \multirow[t]{2}{*}{ Range } & $0-100$ & & Range & 42.86 & \\
\hline & & & & $\begin{array}{r}-100 \\
8457\end{array}$ & \\
\hline Avg. & 60.00 & & Avg. & 84.57 & \\
\hline
\end{tabular}

* Items for which percentage agreement between raters did not meet criteria for acceptability $(60 \%)$ ). 
The final revised tool had only three items which failed to reach an acceptable level of inter-rater agreement: Item 11, paraphrasing and reflecting feelings; Item 29, adverse life events/precipitants; and Item 33, reasons for living.

Item 11 (paraphrasing and reflecting feelings) was from section A 'Interviewing skills'. It could be hypothesised that this item failed for the same reasons that the items on minimal encouragers and open questioning approached inadequate agreement. These questions required the raters to quantify the extent to which clinicians used counselling skills consistently over the length of the interview. The process of converting this estimate into a dichotomous rating (generally uses this technique or inconsistently uses this technique) created too much error. Improved consistency has been indicated by Morriss et al. (1999) when using a quantitative tool. Future revision of the CSRAC could include recording of absolute numbers of utterances from the clinician that: checked meaning, reflected feeling, gave minimal encouragers, and open versus closed questions. This would provide a more objective rating.

The second item which did not meet acceptable inter-rater reliability was Item 29 (adverse life events or precipitants). Disagreement existed over the use of the rating 'partially addressed'. Whilst one rater would indicate that the item had been 'adequately addressed', the other would indicate that it had been only 'partially addressed'. Raters had different requirements for adequate levels of probing and discussion. Moreover raters made different distinctions between precipitating life stressors, for example a death in the family, and perpetuating life stressors, for example current financial difficulty. Therefore there was confusion over the marking criteria for a given rating and the definition of 'adverse life events'. This difficulty is due to the fact that the item is not explicit; unlike for example item 17 'loss of appetite' which had $100 \%$ reliability. The assessment of this item requires subjective judgement on behalf of the rater about the intentions of the clinician.

The third item to obtain insufficient inter-rater reliability was Item 33 (reasons for living). Similarly to the life events item this item was too variable and subjective to allow for consistent ratings. This item was essentially an amalgamation of two concepts from the research and focus group. From the research came explicit reasons for not wanting to suicide (e.g. fear of death) and from the focus group came the protective factor 'areas of enjoyment in life'. The raters agreed that the former concept was not assessed in any interview, but the extent to which the second concept, 'areas of enjoyment' was assessed was complicated by the necessity for rater judgement on the motivations and intentions of the clinicians. A clearer definition of this item could increase reliability. Given the complex and subjective nature of this item, dropping 'areas of enjoyment' from the item is another solution.

It is possible that inter-rater agreement could be improved with the development of a more detailed manual that provides more comprehensive scoring guidelines. This would enhance research application but may limit routine use for evaluation of training and evaluation of practitioner competence.

\section{Limitations}

A major limitation of the current study on interrater reliability was the small number of participants in the vivo interviews. Future research should use larger samples to test reliability across a broader range of sample interviews.

In this study the raters of the tool were also the developers. A great deal of shared understanding of concepts and an exceptional level of familiarity with the tool was possessed by the raters/developers. Despite the developers' attempts to make all definitions and marking criteria explicit, the extent to which novice raters with no prior exposure to the tool would achieve similar results is questionable. Future research should use a number of raters who are unfamiliar with the tool.

We think there may be scope for further development of the content of the tool. While we attempted to ensure that the major risk factors were taken into account in the development of the instrument, we are aware of some factors such as recent discharge from hospital that arguably require inclusion. We are 
also aware that the relative importance of risk factors is a matter of continuing research.

This means that the tool is not ready for routine use in the evaluation of training impact or practitioner competence. We do however think that results obtained so far are promising and suggest it has potential for research application and, with further development, application in training and evaluation of practitioners. While developed specifically for application with clinicians it may, with modifications, also be suitable for use with a range of non-clinical people who receive training in mental health 'first aid'.

Future research should also aim to address questions of the tool's validity. Of particular interest would be the tool's discriminant/criterion validity, which could be established by assessing groups known to differ in risk assessment skills, for example undergraduate students versus crisis team clinicians. Comparison with a similar tool, for example, that from the Morriss et al. (1999) study, to assess convergent validity is also recommended.

The only extant tool we identified in the literature (Morriss et al., 1999) has moderate reliability, untested validity and a ceiling effect when used with mental health professionals. Preliminary results indicate that the tool developed for this study advances the evaluation of risk assessment competency and provides a basis for further development.

\section{Acknowledgements}

The work completed in this study was conducted to meet the partial requirements of the degree of Masters of Occupational Therapy Studies in the Division of Occupational Therapy, The University of Queensland by the last two authors. The authors would like to thank the actor, the focus group participants, and the students who made this work possible.

\section{References}

Aalast, J., Shotts, S., Vitsky, J., Bass, S., Miller, R., Meador, K., \& Morris, J. (1992). Long-term followup of unsuccessful violent suicide attempts: Risk factors for subsequent attempts. Journal of Trauma, $33,457-464$.
Adam, K., Bouckoms, A., \& Streiner, D. (1982). Parental loss and family stability in attempted suicide. Archives of General Psychiatry, 39, 10811085.

American Psychiatric Association (2003). Practice Guidelines for the Assessment of Patients with Suicidal Behaviors. (Last accessed 15 May 2006) http://www.psych.org/psych_pract/treatg/pg/pg_suici dalbehaviors.pdf.

Appleby, L., Morriss, R., Gask, L., Roland, M., Lewis, B., Perry, A., Battersby, L., Colbert, N., Green, G., Amos, T., Davies, L., \& Faragher, B. (2000). An educational intervention for front-line health professionals in the assessment and management of suicidal patients (The STORM Project). Psychological Medicine, 30, 805-812.

Apter, A., Plutchik, R., \& van Praag, H. (1993). Anxiety, impulsivity and depressed mood in relation to suicidal and violent behaviour. Acta Psychiatrica Scandinavica, 87, 1-5.

Barraclough, B., Bunch, J., Nelson, B., \& Sainsbury, P. (1974). A hundred cases of suicide. British Journal of Psychiatry, 125, 355-373.

Beautrais, A. (2003). Subsequent mortality in medically serious suicide attempts: A 5 year followup. Australian and New Zealand Journal of Psychiatry, 37, 595-599.

Cheng, A.T. (1995). Mental illness and suicide. Archives of General Psychiatry, 52, 594-603.

Chynoweth, R., Tonge, J., \& Armstrong, J. (1980). Suicide in Brisbane: A retrospective psychological study. Australian and New Zealand Journal of Psychiatry, 14, 37-45.

Commonwealth Department of Health and Ageing (2000). LIFE - Living is for Everyone: A framework for prevention of suicide and self-harm in Australia. Canberra: Publications Product Unit.

Commonwealth Department of Health and Ageing (2002). National Practice Standards for the Mental Health Workforce. Canberra: Publications Product Unit.

Commonwealth Department of Health and Ageing (2006), National Projects in Mental Health Promotion and Suicide Prevention. http://www.health.gov.au/internet/wcms/publishing.n sf/content/mental-suicide-projects. (Last accessed 15 May, 2006)

Cooper, J., Kapur, N., Webb, R., Lawlor, M., Guthrie, E., \& Appelby, L. (2005). Suicide after deliberate self-harm: A four year cohort study. American Journal of Psychiatry, 162, 297-303.

De Leo, D., Cerin, E., Spathonis, K., \& Burgis, S. (2005). Lifetime risk of suicide ideation and attempts in an Australian community: Prevalence, suicidal 
process, and help-seeking behaviour. Journal of Affective Disorders, 86, 215-224.

Doyle, M. (2003). Developing, delivering and evaluating interprofessional clinical risk training in mental health services. Psychiatric Bulletin, 27, 7376.

Dube, S., Anda, R., Felitti, V., Chapman, D., Williamson, D., \& Giles, W. (2001). Childhood abuse, household dysfunction, and the risk of attempted suicide throughout the life span. Journal of American Medical Association, 286, 3089-3096.

Eddins, C. \& Jobes, D. (1994). Do you see what I see? Patient and clinician perceptions of underlying dimensions of suicidality. Suicide Life: Threatening Behavior, 24, 170-174.

Fenwick, C., Vassilas, C.A., Carter, H., \& Haque, S. M. (2004). Training health professionals in the recognition, assessment and management of suicide risk. International Journal of Psychiatry, 8, 117-121.

Gask, L., Goldberg, D., Lesser, A.L., \& Millar, T. (1988). Improving the psychiatric skills of the general practice trainee: An evaluation of a group training course. Medical Education, 22, 132-138.

Gask, L., Williams, B., \& Harrison, J. (1995). Teaching cognitive-behavioural skills to general practice trainees: A pilot study of training. Primary Care Psychiatry, 1, 201-205.

Harris, E. \& Barraclough, B. (1997). Suicide as an outcome for mental disorders. British Journal of Psychiatry, 170, 205-228.

Hawton, K., Zahl, D., \& Weatherall, R. (2003). Suicide following deliberate self-harm: Long-term follow-up of patients who presented to a general hospital. British Journal of Psychiatry, 182, 537-542.

Heikkinen, E.T., Isometsa, M.E., Marttunen, M.J., Aro, H.M., \& Lonnqvist, J.K. (1995). Social factors in suicide. British Journal of Psychiatry, 167, 747 753.

Hill, C., Thompson, B., \& Williams, E. (1997). A guide to conducting Consensual Qualitative Research. Counseling Psychology, 4, 517-572.

Isacsson, G., Bergmen, U., \& Rich, C.L. (1994). Antidepressants, depression, and suicide: An analysis of the San Diego Study. Journal of Affective Disorders, 32, 277-286.

Isometsa, G., Henriksson, M.M., Aro, H.M., Heikkinen, M.E., Kuopasalmi, K. I., \& Lonnqvist, J.K. (1994). Suicide in major depression. American Journal of Psychiatry, 151(4), 530-536.

Jobes, D. \& Drozd, J. (2004). The CAMS approach to working with suicidal patients. Journal of Contemporary Psychotherapy, 34, 73-85.
Joiner, T., Rudd, D., \& Rajab, H. (1997). The modified scale for suicidal ideation: Factors of suicidality and their relation to clinical and diagnostic variables. Journal of Abnormal Psychology, 106, 260-265.

Joiner, T., Pfaff, J., \& Acres, J. (2002). A brief screening tool for suicidal symptoms in adolescents and young adults in general health settings: Reliability and validity data from the Australian National General Practice Youth Suicide Prevention Project. Behavior Research and Therapy, 40, 471481.

Juhnke, G.A. (1995). Teaching suicide risk assessment to counselor education students. Counselor Education Supervision, 34, 52-57.

Kessler, R., Borgers, G., \& Walters, E. (1999). Prevalence of and risk factors for lifetime attempts in the national co-morbidity survey. Archives of General Psychiatry, 56, 617-626.

Kleespies, P., Deleppo, J., Gallagher, P., \& Niles, B. (1999). Managing suicidal emergencies: Recommendations for the practitioner. Professional Psychology, 30, 454-463.

Linehan, M. \& Goodstein, J. (1983). Reasons for staying alive when you are thinking of killing yourself: The Reasons for Living Inventory. Journal of Consulting Clinical Psychology, 51, 276-286.

Malone, K., Oquendo, M., Haas, G., Ellis, S., Li, S., \& Mann, J. (2000). Protective factors against suicide acts in major depression: Reasons for living. American Journal of Psychiatry, 157, 1084-1088.

Meichenbaum, D. (2005). 35 years working with suicidal patients: Lessons learned. Canadian Psychologist, 46, 64-72.

Morriss, R., Gask, L., Battersby, L., Francheschini, A., \& Robson, M. (1999). Teaching front-line heath and voluntary workers to assess and manage suicidal patients. Journal of Affective Disorders, 52, 77-83.

Neimeyer, R.A. \& Bonnelle, L. (1997). The Suicide Response Inventory: A revision and validation. Death Studies, 21, 59-81.

Nelson-Jones, R. (2003). Basic Counselling Skills: A Helper's Manual. London: Sage Publications.

O’Connor, N., Warby, M., Raphael, B., \& Vassallo, T. (2004). Changeability, confidence, common sense and corroboration: Comprehensive suicide risk assessment. Australian Psychiatry, 12, 352-360.

Paykel, E., Prusoff, B., \& Myers, J. (1975). Suicide attempts and recent life events. Archives of General Psychiatry, 32, 327-333.

Pirkis, J., Burgess, P., \& Jolley, D. (2002). Suicide among psychiatric patients: A case-control study. 
Australian and New Zealand Journal of Psychiatry, 36, 86-91.

Pokorny, A. (1983). Prediction of suicide in psychiatric patients: Report of a prospective study. Archives of General Psychiatry, 40(3), 249-257.

Queensland Government (2003). Reducing Suicide: The Queensland Government Suicide Prevention Strategy 2003-2008. Brisbane: Queensland Government.

Range, L. (2004). The family of instruments that assess suicide risk. Journal of Psychopathology, 27, 133-140.

Rihmer, Z. (1996). Strategies of Suicide Prevention: Focus on heath care. Journal of Affective Disorders, 39, 83-91.

Rogers, J., Lewis, M., \& Subich, L. (2000). Validity of the suicide assessment checklist in an emergency crisis centre. Journal of Counseling Development, 80, 493-502.

Rogers, J. \& Soyka, K. (2004). “One size fits all”: An existential-constructivist perspective on the crisis intervention approach with suicidal individuals. Journal of Contemporary Psychotherapy, 34(1), 722.

Roy, A. (1981). Risk factors for suicide in psychiatric patients. Archives of General Psychiatry, 39, 10891095.

Roy, A. (1983). Family history of suicide. Archives of General Psychiatry 40, 971-974.
Rutz, W. (2001). Mental Health: Diversities, possibilities, shortcomings, challenges. The WHO perspective. European Archives of Psychiatric Clinical Neuroscience, 251(Suppl 2), 3-5.

Schwartz, R.C. \& Rogers, J.R. (2004). Suicide assessment and evaluation strategies: A primer for counselling psychologists. Counseling Psychologist Quarterly, 17, 89-97.

Simon, R. (2004). Assessing and Managing Suicide Risk: Guidelines for clinically based risk management. Washington DC: American Psychiatric Publishing Inc.

Simpson, G., Winstanley, J. \& Bertapelle, T. (2003). Suicide prevention training after traumatic brain injury: Evaluation of a staff training workshop. Journal of Head Trauma Rehabilitation, 18, 445-456.

Tierney, R.J. (1994). Suicide Intervention training evaluation: A preliminary report. Crisis, 15, 69-79.

Victorian Department of Human Services (2003). Evaluation of the Victorian Suicide Prevention Response-Summary report. (Accessed 15 May 2006) www.health.vic.gov.au/mentalhealth/publications/sui cide evaluation/summary.pdf.

Young, M. A., Fogg, L.F., Scheftner, W.A., \& Fawcett, J.A. (1994). Interaction of risk factors in predicting suicide. American Journal of Psychiatry, 151, 434-435.

\section{Appendix 1. Clinician Suicide Risk Assessment Checklist}

\section{Part A: Interviewing Skills}

Please rate the clinician's use of the following interviewing skills on the scale below:

Adequate: Generally adopts this technique Inadequate: Inconsistently adopts this technique

Body language consistent with SOLER:

1. S: Face person squarely to indicate you are with them

2. O: Adopt an open, non-defensive posture i.e. no crossed arms

3. L: Lean towards the client to indicate involvement and availability

4. E: Eye contact maintained, but varied

5. R: Clinician looks comfortable and relaxed

6. Appropriate and responsive facial expressions Which facilitate communication and do not inhibit it.

7. Appropriate use of gestures Gestures with hands or head (e.g. nodding) to encourage disclosure, not distracting or excessive.

8. Non-judgmental response Clinician responds to disclosure in a non-judgemental way.
Adequate Inadequate

A I

A I

A I

A I

A $\quad$ I

A I

A 1

A I 
9. Communication of empathetic understanding

Clinician demonstrates that they are attempting to understand the situation from the client's point of view.

10. Collaborative working relationship established

A I

Does the clinician encourage a shared responsibility for the situation?

11. Paraphrasing and reflecting feelings

A I

Does the clinician check his or her understanding of the client's situation?

Does the clinician reflect the client's feelings?

(must display both to be adequate)

12. Uses minimal encouragers

A $\quad$ I

Small verbal prompts (e.g. 'sure' 'um hum') that facilitate communication and do not inhibit it.

13. Uses open questions where appropriate

Begins with open questions and gradually becomes more closed and specific.

A I

\section{Part B: Risk Assessment}

Please indicate on the scale below whether or not the clinician successfully addressed the following predictors:

Addressed: The clinician addressed this predictor either via direct enquiry or as volunteered by the client. Not addressed: The clinician failed to address this predictor and the client did not volunteer the information.

Partially addressed: The clinician somewhat addressed the predictor. The clinician may have failed to adequately probe for sufficient detail and/or there was some ambiguity of its meaning/context to the client.

History

Addressed Not addressed Partially

14. Previous and current non-depressive psychiatric history

Does the clinician address presence or absence of past and present psychiatric symptoms or diagnosis?

15. Past episodes of depression?

Did clinician establish past episodes of depression AND treatments for these?

If clinician enquires about depression only, a rating of partial is administered.

16. Suicidality of somebody close

Friend who has recently suicided or immediate family member who has ever suicided?

17. Family history

Does the clinician enquire about how the client feels about their current family relationships and childhood?

If one is discussed give a rating of partial, if both are discussed give a rating of addressed.

\section{Mental state examination}

18. Presence of depression

Does the clinician establish if the client currently has depressed mood? Severity of depressed mood:

19. Hopelessness

Does the clinician determine client's thoughts concerning hope for improvement in the future?

20. Worthlessness

Does the clinician attempt to understand how the client perceives themselves in terms of worth of self and worth to others?

21. Other: Appetite/weight loss, sleep/insomnia, reduced pleasure/reduced libido, poor concentration and/or memory

Does the clinician address one or more of the above depressive symptoms?

22. Impact on functioning

Does the clinician attempt to address the impact of the client's symptoms on current functioning (work and/or studies, leisure, self-care)?

If one is discussed, then to be rated as addressed States \& traits:

23. Drug \& alcohol abuse

Does the clinician determine type, frequency, effect and after effects?

If only one is elicited then should be rated as partial

24. Impulsivity

Does the clinician attempt to determine whether the client has completed impulsive acts in the past or has demonstrated impulsive behaviour? i.e. tend to do things without considering the consequences of their actions. 
King et al.

Self-harm/suicidal behaviour

Addressed Not addressed Partially

25. Previous self-harm

Did the clinician determine previous non-lethal self-harm, e.g. burning, or cutting?

26. Suicide desire and ideation

Did the clinician attempt to address presence or absence of suicidal ideation, and if present the nature of the ideation? If the clinician does not follow-up ambiguous statements a rating of partial is given

If the client expresses suicidal ideation in the past OR present Q27 applies. If no, then does not apply.

27. Previous suicidal behaviour

Did the clinician determine previous attempts or passive attempts?

If the client expresses that they have never attempted and never will attempt suicide then Q28 does not apply. e.g. "I would never really do it"

28. Level of intent, resolved plans and preparation

Did the clinician address the client's sense of desperation, opportunity and capability to complete the act?

Did the clinician establish presence of availability of means, specificity of plan, preparations, and making final plans, e.g. taking care of unfinished business and/or giving away possessions?

\section{Personal \& situational stressors}

29. Adverse life events/precipitants

Common stressors include legal problems, financial problems, family relationship difficulty, interpersonal crisis, feels as though burden on others, recent loss.

Does the clinician attempt to understand the client's situation and presence of recent stressful life events?

Does the clinician attempt to understand these in terms of its meaning to the client?

If the clinician establishes stressors at the onset of problem and not current stressors the rating should be partial. If current stressors are discussed the rating should be addressed.

30. Physical illness

Does the clinician enquire about significant physical illness and its impact on the client?

31. Social isolation

Does the clinician attempt to determine the extent of the client's support network, including friends, family and others?

32. Coping skills/coping skill exhaustion

Does the clinician attempt to determine the presence or absence of coping skills?

Does the clinician attempt to determine if these are currently working effectively or if they have been exhausted?

If only one of these is addressed the rating should be partial.

\section{Protective factors}

33. Reasons for living

Did the clinician give the client the opportunity to identify presence OR absence of factors which they felt may protect them against the act of suicide?

Discussion of strengths/areas of enjoyment is a rating of partial, explicit 'reasons for living' is rated as addressed. Common reasons for living include: Child living at home under the age of 18, social support, negative beliefs about suicide, fear of death or the act itself, and religious faith.

\section{Part C: Intervention Planning}

\section{Plan constructed}

Did the clinician attempt to formulate the beginning of an operational management plan?

If a plan has been discussed but not concretely established a rating of partial should be made.

35. Client agreement

Does the client agree with the plan?

36. Client input

Did the clinician seek input from the client in terms of what they wanted to achieve and/or how to achieve it?

37. Follow-up

Did the clinician establish a date for follow-up for re-assessment and monitoring of the plan?

Follow-up may be a referral to another health professional. If an agreed upon time/date is not determined the rating should be partial.

38. Appropriateness of plan

Does the intensity of the plan reflect the level of risk ascertained?

If the plan has not been operationalised the highest rating allowable is partial.

39. Plan is feasible

Is the plan practical given the context and capabilities of the client? 